\title{
A Study on Urban Road Traffic Safety Based on Matter Element Analysis
}

\author{
Qizhou Hu, ${ }^{1}$ Zhuping Zhou, ${ }^{1}$ and Xu Sun ${ }^{2}$ \\ ${ }^{1}$ School of Automation, Nanjing University of Science and Technology, Nanjing 210094, China \\ ${ }^{2}$ Institute of Transportation Engineering, Tsinghua University, Beijing 100084, China \\ Correspondence should be addressed to Zhuping Zhou; jzp19850926@163.com
}

Received 11 September 2014; Revised 30 November 2014; Accepted 8 December 2014; Published 22 December 2014

Academic Editor: Yongjun Shen

Copyright ( 2014 Qizhou Hu et al. This is an open access article distributed under the Creative Commons Attribution License, which permits unrestricted use, distribution, and reproduction in any medium, provided the original work is properly cited.

\begin{abstract}
This paper examines a new evaluation of urban road traffic safety based on a matter element analysis, avoiding the difficulties found in other traffic safety evaluations. The issue of urban road traffic safety has been investigated through the matter element analysis theory. The chief aim of the present work is to investigate the features of urban road traffic safety. Emphasis was placed on the construction of a criterion function by which traffic safety achieved a hierarchical system of objectives to be evaluated. The matter element analysis theory was used to create the comprehensive appraisal model of urban road traffic safety. The technique was used to employ a newly developed and versatile matter element analysis algorithm. The matter element matrix solves the uncertainty and incompatibility of the evaluated factors used to assess urban road traffic safety. The application results showed the superiority of the evaluation model and a didactic example was included to illustrate the computational procedure.
\end{abstract}

\section{Introduction}

A major concern in the world today is the continued improvement of traffic safety. At present, urban road traffic safety has become a prominent social problem, causing large economic loss and human casualties. Considering all these issues, the study of urban road traffic safety has become more and more important [1]. The purpose of traffic safety evaluation of urban road is to make comparative satisfaction judgment, find out hidden safety trouble, take various corresponding measures, and reduce accident occurrence. Although much effort has been placed into improving these issues, an efficient and effective method has yet to be developed. The traffic safety system is extremely complex and many influential factors exist. Because of their uncertainty, it is difficult to quantify these factors and qualitative disposals are usually adopted. To evaluate urban road traffic safety, Wang et al. selected several factors of primary influence to evaluate the index, including road surface condition, traffic isolation, monitor establishment, congestion level, and accident ratio and tunnel establishment [2]. Taubman et al. implemented an evaluation by qualitative analysis [3]. The following flaws must be considered in this analysis: (1) the final appraisal cannot determine whether the urban road traffic safety condition as "good" or "bad"; (2) appraisal is based on an insufficient scientific nature, hindering its feasibility, and commensurability. There are no standard ways of assessing urban road traffic safety; therefore, scientific methods for such assessments are still needed.

During the past decade, the theory of matter element analysis has developed in numerous directions. Creating a quantitative appraisal model with quantitative evaluation provides the main study subject. However, there are many factors influencing urban road traffic safety. The actual conditions are quite complicated. It is very difficult to quantify the appraisal indices with specific functions and evaluate urban road traffic safety with a certain qualitative model [4]. Many evaluating processes take place in an environment in which the information is not precisely known [5]. Evaluation makers may have vague knowledge on the preference degrees of one alternative over another [6]. They cannot estimate their preference through exact numerical values, but through interval numbers. Based on the concept of interval numbers, a new method to solve the traffic safety problems is proposed in this paper. First, we define the concept of interval numbers and state the operational laws. Second, based on the concept 
TABLE 1: Quantification of the evaluation index.

\begin{tabular}{lccccc}
\hline Criterion & Excellent & Good & Medium & General & Poor \\
\hline Evaluation interval & {$[0.9,1]$} & {$[0.8,0.9]$} & {$[0.7,0.8]$} & {$[0.6,0.7]$} & {$[0.0,0.6]$} \\
\hline
\end{tabular}

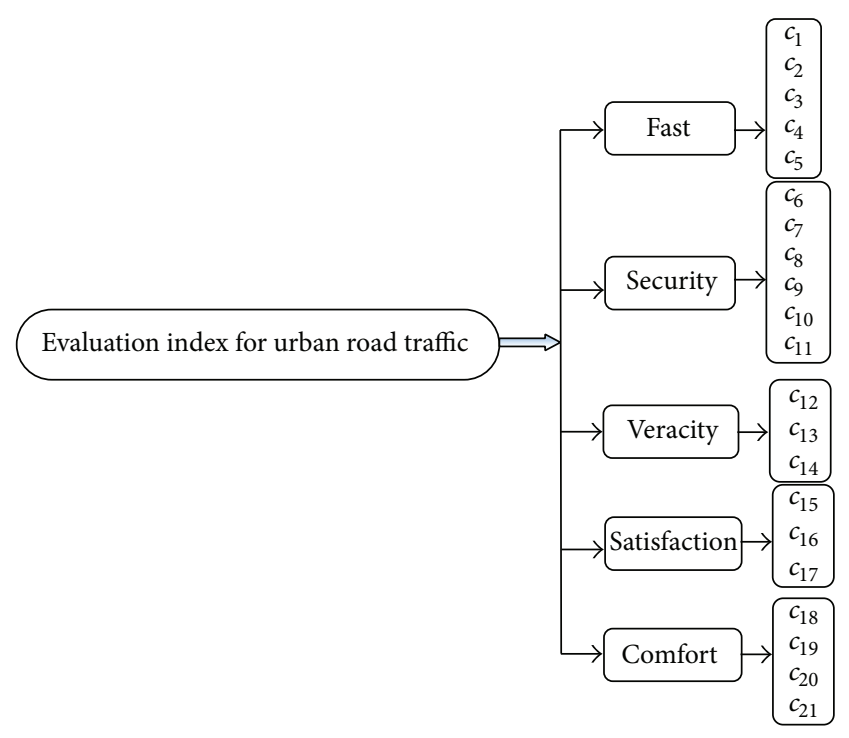

Figure 1: The evaluation system for urban road traffic safety.

of matter element, this paper offers a new method to solve the traffic safety problems through interval numbers. Finally, an example is given to demonstrate the feasibility and superiority of our proposed new method.

\section{The Evaluation Index System for Urban Road Traffic Safety}

The evaluation of urban road traffic safety is multifield and multisubject. The evaluation index selection must meet two standards: (1) the indices must enable the evaluation system to reflect the actual situation of urban road traffic safety completely and accurately and (2) the evaluation index system of urban road traffic safety must be as simple as possible.

2.1. The Traffic Safety Evaluation System of Urban Road. In order to choose the evaluation indices correctly, we must make an accurate analysis on the traffic safety of urban road systems. Then the indices will reflect various influences objectively. Each evaluation index is selected by using the qualitative comparison method. For the convenience of the analysis of the computation, we obtain urban road safe evaluation target system through the generalized analysis of urban road traffic safety, as is shown in Figure 1.

$c_{1}$ is the road network connectivity, $c_{2}$ is the density of road network, $c_{3}$ is chuckles level of joint, $c_{4}$ is the speed of road network, $c_{5}$ is road network volume, $c_{6}$ is apparent distance, $c_{7}$ is traffic segregation, $c_{8}$ is protection facilities, $c_{9}$ is lighting facilities, $c_{10}$ is tunnel facilities, $c_{11}$ is monitoring facilities, $c_{12}$ is guide facilities, $c_{13}$ is information system, $c_{14}$ is weather forecast information facilities, $c_{15}$ is pavement behavior, $c_{16}$ is traffic accident rate, $c_{17}$ is landscape along the line, $c_{18}$ is mileage saturation rate, $c_{19}$ is traffic congestion level, $c_{20}$ is smooth-riding quality, and $c_{21}$ is geographical features.

\subsection{The Evaluation Indices of Urban Road Traffic Safety} Quantified. Evaluation indices are classified into quantitative and qualitative indices. Each index has different meanings with different corresponding values [7]. In order to allow a common degree between each evaluation index of urban road traffic safety, we must carry standardized processing on the indices.

(1) The calculation method for the quantitative index of urban road traffic safety is as follows.

After the evaluation index is determined, we can then state the property value of the index of urban road traffic safety by either booking for statistical data, or deriving calculation.

(2) The calculation method for the qualitative index of urban road traffic safety is as follows.

It utilizes (an unclear) fuzzy function to complete the quantitative disposal for the qualitative index of urban road traffic safety. It imposes (an unclear) fuzzy transformation theory and maximal membership principle to set up quantitative evaluation conclusion without subjective factor [8]. Here it adopts (ambiguous) fuzzy language in mathematics that carry through standardization. It can be shown in Table 1.

2.3. The Grading Standards of the Evaluation Index of Urban Road Traffic Safety. Practically in evaluation making, the evaluation makers give the observed values of the index in the form of linguistic numbers, such as "bad, common, good, better, best," or "low, common, high, higher, highest. ." [9]. The main foundation of this paper is the application of rulebased decision-making. Since differences exist in the knowledge frame, experience level, status and individual preference for the evaluation makers, and incomplete, inadequate information is present in decision-making problems, so the observed values of the index given by the evaluation makers have a certain degree of grey area [10].

The factors mentioned above are divided into 5 grades (grade I, grade II, grade III, grade IV, and grade V) according to their influence on urban road traffic safety. Grade I indicates great safety influence in urban traffic; grade II indicates that safety influence exists on a small scale and has a large potential for urban traffic problems; grade III indicates safety influence of urban traffic exists in a large scale, but it still has some potentials; grade IV indicates that safety influence of urban traffic is near to the extremity; grade $\mathrm{V}$ indicates that 
TABLE 2: The grading standards of the evaluating index.

\begin{tabular}{|c|c|c|c|c|c|}
\hline \multirow{2}{*}{ Evaluation index } & Grade I & Grade II & Grade III & Grade IV & Grade V \\
\hline & Excellent & Good & Medium & General & Poor \\
\hline$c_{1}$ & $0 \sim 0.2$ & $0.2 \sim 0.4$ & $0.4 \sim 0.6$ & $0.6 \sim 0.8$ & $>0.8$ \\
\hline$c_{2}\left(\mathrm{~km} / \mathrm{km}^{2}\right)$ & $0 \sim 2.5$ & $2.5 \sim 3.0$ & $3.0 \sim 3.5$ & $3.5 \sim 4.5$ & $>4.5$ \\
\hline$c_{3}$ & $0 \sim 0.5$ & $0.5 \sim 0.6$ & $0.6 \sim 0.7$ & $0.7 \sim 0.9$ & $>0.9$ \\
\hline$c_{4}(\mathrm{~km} / \mathrm{h})$ & $0 \sim 10$ & $10 \sim 20$ & $20 \sim 30$ & $30 \sim 40$ & $>40$ \\
\hline$c_{5}($ standard units $/ \mathrm{km})$ & $>5.5$ & $4.5 \sim 5.5$ & $3.5 \sim 4.5$ & $2.5 \sim 3.5$ & $0 \sim 2.5$ \\
\hline$c_{6}(\mathrm{~m})$ & $0 \sim 5$ & $5 \sim 10$ & $10 \sim 15$ & $15 \sim 20$ & $>20$ \\
\hline$c_{7}$ & $0 \sim 0.6$ & $0.6 \sim 0.7$ & $0.7 \sim 0.8$ & $0.8 \sim 0.9$ & $>0.9$ \\
\hline$c_{8}$ & $0 \sim 0.6$ & $0.6 \sim 0.7$ & $0.7 \sim 0.8$ & $0.8 \sim 0.9$ & $>0.9$ \\
\hline$c_{9}$ & $0 \sim 0.6$ & $0.6 \sim 0.7$ & $0.7 \sim 0.8$ & $0.8 \sim 0.9$ & $>0.9$ \\
\hline$c_{10}$ & $0 \sim 0.6$ & $0.6 \sim 0.7$ & $0.7 \sim 0.8$ & $0.8 \sim 0.9$ & $>0.9$ \\
\hline$c_{11}$ & $0 \sim 0.6$ & $0.6 \sim 0.7$ & $0.7 \sim 0.8$ & $0.8 \sim 0.9$ & $>0.9$ \\
\hline$c_{12}$ & $0 \sim 0.6$ & $0.6 \sim 0.7$ & $0.7 \sim 0.8$ & $0.8 \sim 0.9$ & $>0.9$ \\
\hline$c_{13}$ & $0 \sim 0.6$ & $0.6 \sim 0.7$ & $0.7 \sim 0.8$ & $0.8 \sim 0.9$ & $>0.9$ \\
\hline$c_{14}$ & $0 \sim 0.6$ & $0.6 \sim 0.7$ & $0.7 \sim 0.8$ & $0.8 \sim 0.9$ & $>0.9$ \\
\hline$c_{15}$ & $0 \sim 0.6$ & $0.6 \sim 0.7$ & $0.7 \sim 0.8$ & $0.8 \sim 0.9$ & $>0.9$ \\
\hline$c_{16}(\%)$ & $>0.05$ & $0.04 \sim 0.05$ & $0.03 \sim 0.04$ & $0.02 \sim 0.03$ & $0 \sim 0.02$ \\
\hline$c_{17}$ & $0 \sim 0.6$ & $0.6 \sim 0.7$ & $0.7 \sim 0.8$ & $0.8 \sim 0.9$ & $>0.9$ \\
\hline$c_{18}(\%)$ & $>0.8$ & $0.7 \sim 0.8$ & $0.6 \sim 0.7$ & $0.5 \sim 0.6$ & $0 \sim 0.5$ \\
\hline$c_{19}$ & $>0.8$ & $0.7 \sim 0.8$ & $0.6 \sim 0.7$ & $0.5 \sim 0.6$ & $0 \sim 0.5$ \\
\hline$c_{20}$ & $0 \sim 0.2$ & $0.2 \sim 0.4$ & $0.4 \sim 0.6$ & $0.6 \sim 0.8$ & $>0.8$ \\
\hline$c_{21}$ & $0 \sim 0.6$ & $0.6 \sim 0.7$ & $0.7 \sim 0.8$ & $0.8 \sim 0.9$ & $>0.9$ \\
\hline
\end{tabular}

safety influence of urban traffic is extreme. The evaluating standards can be seen in Table 2 .

\section{Matter Element Analysis Model of Urban Road Traffic Safety}

In this section, basic terminologies and notations are introduced, necessarily to understand the subsequent results. The evaluation model, in absence of more precise insight, is based on various assumptions, some of which are simplified with respect to reality. At this stage, it is difficult to assess the validity and reliability of the evaluation model. It provides, however, a practical but founded and transparent method to address the problem of assessment of urban road traffic safety when only incomplete data are available, by enabling comparative analysis of urban road traffic safety with different nature. The evaluation model is also a valuable tool used to analyze the underlying mechanisms of traffic accident. The assumptions and resulting uncertainties concern the qualitative and quantitative analysis of the relationships between humans, vehicles, and road.

3.1. Basic Knowledge of Interval Numbers. Interval computation is a popular way to treat uncertainties in data measurements. Instead of exact values, we compute with real intervals, which are supposed to involve all gauging errors. An interval numbers is defined as $[\bar{A}]=\left[a_{1}, a_{2}\right]$, where $0 \leq a_{1} \leq a_{2}$ are fixed. $a_{1}$ is the lower element in interval $\left[a_{1}, a_{2}\right] . a_{2}$ is the upper elements in interval $\left[a_{1}, a_{2}\right]$.
For the convenience of calculating interval numbers, some operational laws of interval numbers are introduced as follows [11]. Let $[\bar{A}]=\left[a_{1}, a_{2}\right]$ and $[\bar{B}]=\left[b_{1}, b_{2}\right]$ be interval numbers; then the basic operations on interval numbers are as follows:

(1) $\left[a_{1}, a_{2}\right] \pm\left[b_{1}, b_{2}\right]=\left[a_{1} \pm b_{1}, a_{2} \pm b_{2}\right]$;

(2) $\left[a_{1}, a_{2}\right] \cdot\left[b_{1}, b_{2}\right]=\left[a_{1} \cdot b_{1}, a_{2} \cdot b_{2}\right],\left[a_{1}, a_{2}\right] \div\left[b_{1}, b_{2}\right]=$ $\left[a_{1} / b_{1}, a_{2} / b_{2}\right]\left(b_{1} \neq 0, b_{2} \neq 0\right)$;

(3) if $k \geq 0$, then $k\left[a_{1}, b_{1}\right]=\left[k a_{1}, k b_{1}\right]$, if $k<0$, then $k\left[a_{1}, b_{1}\right]=\left[k b_{1}, k a_{1}\right]$.

3.2. Basic Model of Matter Element. If the name, character, and value of a matter are provided, the three ordinal elements $R=(N, C, V)$ are called a matter element $[12,13]$. There are three basic elements of a matter element, for example, the matter's name $N$, the character $C$, and the value $V$. If a matter $N$ has some characters and can be described by $n$ characters $c_{1}, c_{2}, \ldots, c_{n}$ and its value $x_{1}, x_{2}, \ldots, x_{n}$, thus $R=(N, C, V)$ is called an $n$-dimension matter element.

(1) Matter element matrix [14] is as follows:

$$
R(\text { matter })=\left[\begin{array}{ccc}
N & c_{1} & x_{1} \\
& c_{2} & x_{2} \\
& \vdots & \vdots \\
& c_{n} & x_{n}
\end{array}\right] .
$$

(2) Matter element matrix of classical field object is as follows. 
Given $m$ evaluating degrees as $N_{1}, N_{2}, \ldots, N_{m}$, the following matter element matrix of classical field object can be built as follows:

$$
R_{i}(\text { classical })=\left[\begin{array}{ccc}
N_{i} & c_{1} & {\left[\bar{X}_{i 1}\right]} \\
& c_{2} & {\left[\bar{X}_{i 2}\right]} \\
& \vdots & \vdots \\
& c_{n} & {\left[\bar{X}_{i n}\right]}
\end{array}\right],
$$

where $N_{i}$ is standard object and $i=1,2, \ldots, m,\left[\bar{X}_{i j}\right]=$ $\left[a_{i j}, b_{i j}\right]$ is the value range of $c_{j}(j=1,2, \ldots, n)$ over standard object $N_{i}$.

(3) Matter element matrix of section field object is as follows.

According to formula (2), we can construct its section filed

$$
R_{i}(\text { section })=\left[\begin{array}{ccc}
N_{p i} & c_{1} & {\left[\bar{X}_{p i 1}\right]} \\
& c_{2} & {\left[\bar{X}_{p i 2}\right]} \\
& \vdots & \vdots \\
& c_{n} & {\left[\bar{X}_{p i n}\right]}
\end{array}\right]
$$

where $N_{p i}$ is section object, $\left[\bar{X}_{p i j}\right]=\left[a_{p i j}, b_{p i j}\right]$ is the interval range of the measured value of the character $C$ about section field object, and $\left[\bar{X}_{p i j}\right] \subset\left[\bar{X}_{i j}\right](j=1,2, \ldots, n)$.

\subsection{The Comprehensive Appraisal Model of Urban Road Traffic} Safety. Urban road traffic safety is a crucial subject. Determining the safety levels may help prevent future traffic accidents in the city. But it is not an easy task. Many parameters have considerable effects on the phenomenon. Determining safety levels has a degree of uncertainty. This study deals with determination of city safety levels using order matter element. The analysis approach considers accident types and effective factors on accident occurrence. Geometrical and physical conditions, traffic volumes, average speeds, and average accident rates at around traffic safety are considered as effective factors in city. Matter element matrix is calculated using these parameters. Validation of matter element analysis approach is tested by truth-value methods, and encouraging results are obtained.

Step 1 (matter element matrix of urban road traffic safety). For the estimated object $P_{i}$, the prediction result of urban road traffic safety can be represented by the following matter element matrix of urban road traffic safety:

$$
R\left(P_{i}\right)=\left[\begin{array}{ccc}
P_{i} & c_{1} & x_{i 1} \\
& c_{2} & x_{i 2} \\
& \vdots & \vdots \\
& c_{n} & x_{i n}
\end{array}\right] .
$$

Step 2 (the distance calculation of matrix for urban road traffic safety).

Definition 1. If $\left[\bar{X}_{i j}\right]=\left[a_{i j}, b_{i j}\right]$, then the relating coefficient of urban road traffic safety is

$$
\begin{gathered}
\rho\left(x_{i j},\left[\bar{X}_{i j}\right]\right)=\left|x_{i j}-0.5\left(a_{i j}+b_{i j}\right)\right|-0.5\left(b_{i j}-a_{i j}\right), \\
\rho\left(x_{i j},\left[\bar{X}_{p j}\right]\right)=\left|x_{i j}-0.5\left(a_{p j}+b_{p j}\right)\right|-0.5\left(b_{p j}-a_{p j}\right),
\end{gathered}
$$

where $i=1,2, \ldots, m$ and $j=1,2, \ldots, n$.

Step 3 (relation function about urban road traffic safety).

Definition 2. If $\left[\bar{X}_{i j}\right]=\left[a_{i j}, b_{i j}\right]$, then the relating function of urban road traffic safety is

$$
K\left(x_{i j}\right)= \begin{cases}-\rho\left(x_{i j},\left[\bar{X}_{i j}\right]\right) \cdot\left|\left[\bar{X}_{i j}\right]\right|^{-1}, & x_{i j} \in\left[\bar{X}_{i j}\right] \\ \rho\left(x_{i j},\left[\bar{X}_{i j}\right]\right) \cdot\left[\rho\left(x_{i j},\left[\bar{X}_{p j}\right]\right)-\rho\left(x_{i j},\left[\bar{X}_{i j}\right]\right)\right]^{-1}, & x_{i j} \notin\left[\bar{X}_{i j}\right],\end{cases}
$$

where $i=1,2, \ldots, m$ and $j=1,2, \ldots, n$.

Step 4 (entropy-weight coefficient of the index system of urban road traffic safety). Traditional AHP [15] focuses on the knowledge and experience of experts as well as their wishes and preferences of decision-making thus unable to overcome the subjective and arbitrary mistakes. Entropy-weight can fully exploit the information implied in the original data itself, but it lacks the knowledge and experience from experts and decision-makers. So together, the two methods complement each other. The original information contains quality and quantity, which make it possible to weigh the information in the decision work. Let us denote by

$$
h_{i j}=-\frac{1}{\ln m} \sum_{i=1}^{m} x_{i j} \ln x_{i j}
$$

where $i=1,2, \ldots, m$ and $j=1,2, \ldots, n$ and set $x_{i j} \ln x_{i j}=0$ while $x_{i j}=0$.

Then, the weight value of urban road traffic safety can be calculated by (8)

$$
w_{i j}=\left(1-h_{i j}\right) \cdot\left(m-\sum_{j=1}^{m} h_{i j}\right)^{-1} .
$$

Step 5 (the relating degree of urban road traffic safety). According to the weight of the appraisal index value $w_{i j}$ and the relation function $K\left(x_{i j}\right)$, the comprehensive appraisal value of urban road traffic safety is obtained as follows:

$$
K\left(P_{i}\right)=\sum_{j=1}^{n} w_{i j} K\left(x_{i j}\right) \quad(i=1,2, \ldots, m) .
$$




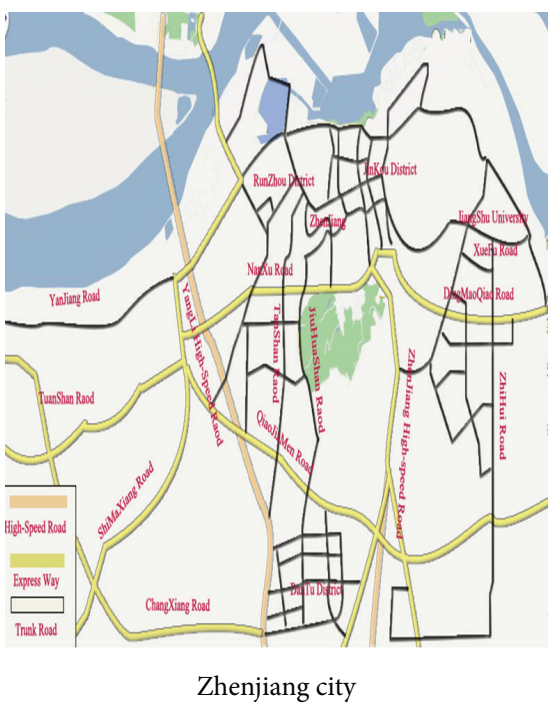

(a)

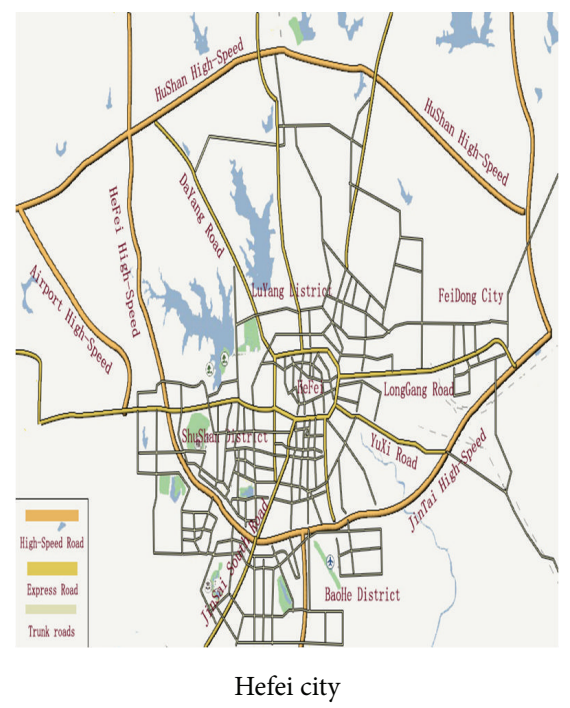

(b)

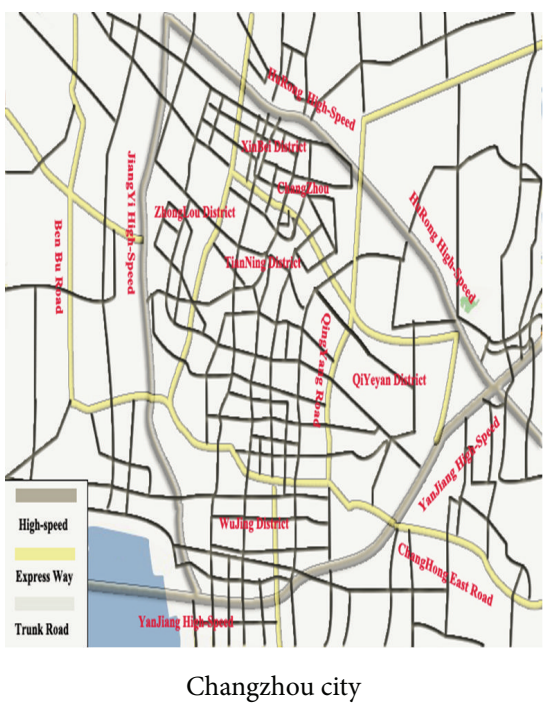

(c)

FIGURE 2: The map for three cities.

Then $K\left(P_{i}\right)$ is defined as the relating degree of the evaluated matter $P_{i}$ with regard to $i$ 's grade. According to the principle of the maximum dependence, the relating function value $K\left(P_{i}\right)$ is maximized:

$$
K_{i 0}=\max _{1 \leq i \leq m}\left\{K\left(P_{i}\right)\right\} .
$$

Hence, the estimating matter $P_{i}$ should belong to the $i_{0}$ 's grade by CAI Wen.

Appraisal set is a set which we get appraisal conclusion for appraisal target $[16,17]$. The traffic safety comprehensive appraisal grade can be obtained in appraisal set $F$. Appraisal set $F=\{$ the first grade (i.e., "Excellent"), the second grade (i.e., "Good"), the third grade (i.e., "Medium"), the fourth grade (i.e., "General"), the fifth grade (i.e., "Poor")\}.

Step 6 (the appraisal standardization of urban road traffic safety). Based on the comprehensive appraisal value $K\left(P_{i}\right)$ and the appraisal grade standard of urban road traffic safety, the comprehensive appraisal grade of urban road traffic safety is analyzed and hence obtained. This value quantitatively reflects urban road traffic safety "good" or "bad" and provides the scientific basis of decision-making for urban road construction. The greater the comprehensive appraisal value $K\left(P_{i}\right)$, the better the urban road traffic safety in the monitoring period. In counterpart, the smaller the comprehensive appraisal value $K\left(P_{i}\right)$, the worse the urban road traffic safety in the monitoring period.

\section{Study on the Measures of Urban Road Traffic Safety}

It is necessary and desired to solve the problems of the urban road traffic safety by using comprehensive management knowledge from all sides. In the light of features of the urban road traffic safety, the analysis results achieved in the paper suggest the following: (i) on one hand, it is essential to improve the supervision and control measures of traffic safety. Urban road has been installed with advanced and modernized facilities, among which traffic control system is the key approach for urban road traffic management [18]. The traffic control system meets the needs of traffic control, monitoring, accessibility, and communication. Under the circumstances of adverse weather and traffic jams, the system can lead drivers to the safe drive and provide traffic supervision and a control system. (ii) On the other hand, vigorous measures are adopted to develop intelligent transportation systems. Intelligent systems are the front topics in the field of transportation all over the world. Intelligent transportation systems are capable of improving traffic capacity and safety of existing networks. They represent the development orientation of traffic science [19]. When the traffic science and technology have great breakthroughs and developments, intelligent transportation technologies can decrease traffic congestion and traffic accident, heighten the labor productivity, intensify the international competition, and increase new industry in future. By means of high-tech, computer science, and communication technology, people can improve the systematic engineering of electromechanical devices for communication, charges, and supervision and monitoring which can make intelligent urban road grow. Developing intelligent urban road can resolve traffic jams, ensure driving safety, and improve service quality. In a word, it can represent the characteristics of modernized urban road [20].

\section{Application Analyses}

In order to carry out rational appraisal to urban road traffic safety in three cities, for example, Zhenjiang city, Hefei city, and Changzhou city, in China are shown in Figure 2, urban 
road on-the-spot from June to July in 2010 was surveyed, and a lot of effective data about the traffic safety were obtained which could quantitatively and comprehensively evaluate the road safety of the three cities. The specific values $x_{i j}$ are shown in Table 3.

Step 1. Determine matter element matrix $R$ and section filed $R_{i}$ of urban road traffic safety.

According to Table 1, take value region corresponding to the evaluating criterion of grades $\mathrm{I}-\mathrm{V}$ as the sutra filed $R_{i}$.

(1) For the first city (i.e., "Zhenjiang city in China")

$$
R_{1} \text { (matter) }=\left[\begin{array}{ccc}
N & c_{1} & 2.81 \\
& c_{2} & 6.72 \\
& c_{3} & 0.71 \\
& c_{4} & 36.7 \\
& c_{5} & 3.80 \\
& c_{6} & 6.74 \\
c_{7} & 0.62 \\
c_{8} & 0.65 \\
c_{9} & 0.77 \\
c_{10} & 0.68 \\
c_{11} & 0.61 \\
c_{12} & 0.78 \\
c_{13} & 0.61 \\
c_{14} & 0.73 \\
c_{15} & 0.68 \\
c_{16} & 0.035 \\
c_{17} & 0.68 \\
c_{18} & 0.75 \\
c_{19} & 0.57 \\
c_{20} & 0.68 \\
c_{21} & 0.78
\end{array}\right]
$$

(2) For the second city (i.e., "Hefei city in China")

$$
R_{2} \text { (classical) }=\left[\begin{array}{ccc}
N & c_{1} & 3.12 \\
& c_{2} & 6.91 \\
c_{3} & 0.54 \\
c_{4} & 53.7 \\
c_{5} & 3.17 \\
& c_{6} & 6.65 \\
c_{7} & 0.71 \\
c_{8} & 0.67 \\
c_{9} & 0.61 \\
c_{10} & 0.55 \\
c_{11} & 0.63 \\
c_{12} & 0.58 \\
c_{13} & 0.53 \\
c_{14} & 0.71 \\
c_{15} & 0.54 \\
c_{16} & 0.037 \\
c_{17} & 0.61 \\
c_{18} & 0.73 \\
c_{19} & 0.68 \\
c_{11} & {[0.0,0.6]} \\
c_{12} & {[0.0,0.6]} \\
c_{13} & {[0.0,0.6]} \\
c_{13} & {[0.73} \\
c_{13} & {[0.03,0.04]} \\
c_{10} & {[0.7,0.8]} \\
c_{19} & {[0.6,0.8]} \\
c_{21} & 0.69
\end{array}\right]
$$




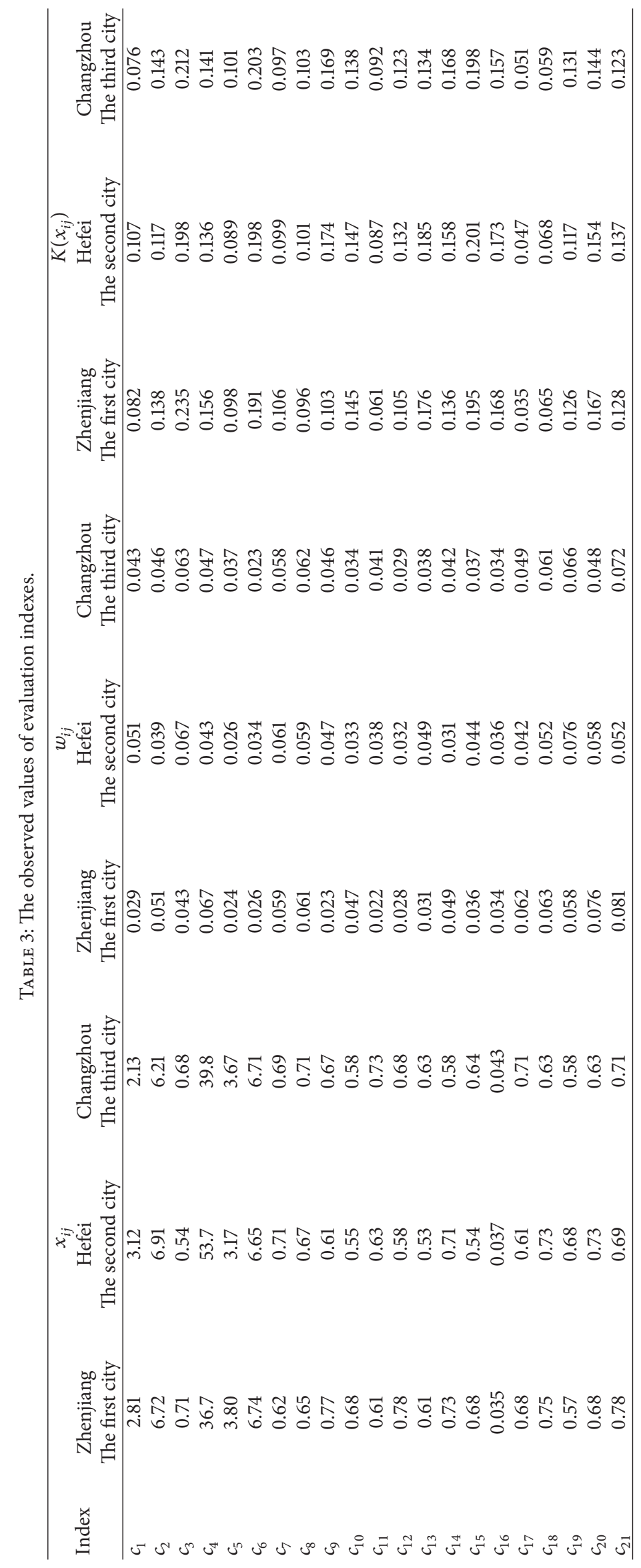


TABLE 4: The evaluating result and the synthetic relating degree in each city.

\begin{tabular}{|c|c|c|c|c|c|c|c|}
\hline \multirow{3}{*}{ Name } & & \multicolumn{5}{|c|}{ The grade of urban road traffic safety } & \multirow{3}{*}{ The evaluation result } \\
\hline & & Very safe & Safe & Approximately & Unsafe & Very safe & \\
\hline & & Level 1 & Level 2 & Level 3 & Level 4 & Level 5 & \\
\hline The first city & Zhenjiang & 0.1127 & 0.2732 & 0.3013 & 0.1889 & 0.2229 & Level 3 \\
\hline The second city & Hefei & 0.1237 & 0.1721 & 0.2136 & 0.2681 & 0.2335 & Level 4 \\
\hline The third city & Changzhou & 0.1711 & 0.1882 & 0.2027 & 0.2032 & 0.2362 & Level 2 \\
\hline
\end{tabular}

(3) For the third city (i.e., "Changzhou city in China")

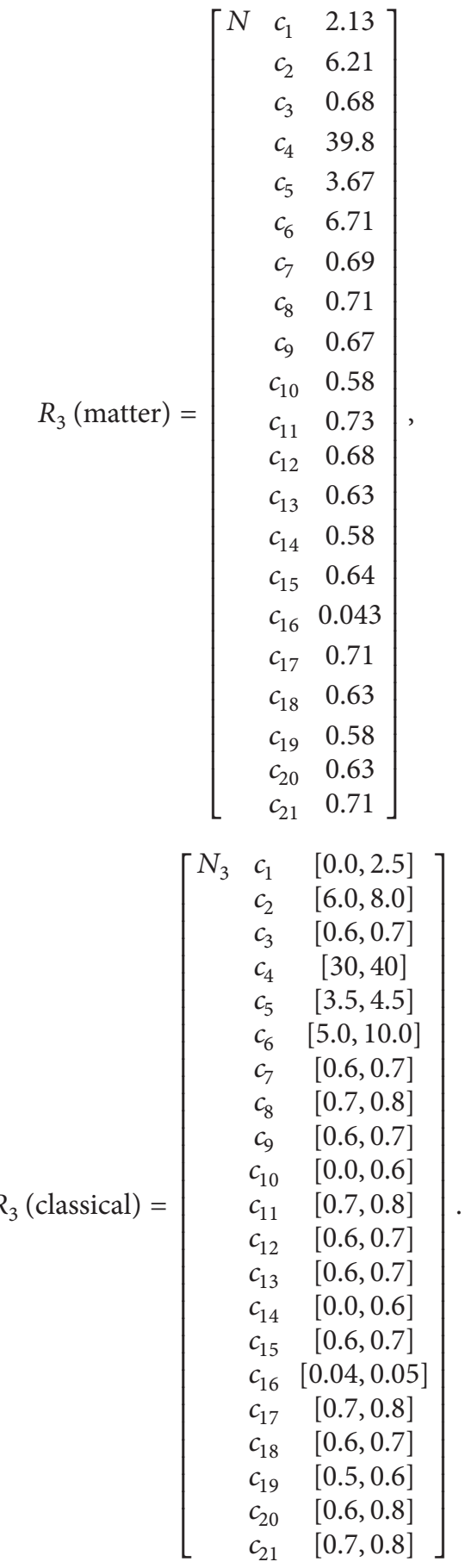

Step 2. Determine the section field $R_{p}$ of urban road traffic safety:

$$
R_{p}(\operatorname{section})=\left[\begin{array}{ccc}
N_{p} & c_{1} & {[0.0,1.0]} \\
& c_{2} & {[0.0,10.0]} \\
& c_{3} & {[0.0,1.0]} \\
& c_{4} & {[0.0,1.0]} \\
& c_{5} & {[0.0,10.0]} \\
& c_{6} & {[0.0,1.0]} \\
& c_{7} & {[0.0,1.0]} \\
& c_{8} & {[0.0,1.0]} \\
& c_{9} & {[0.0,1.0]} \\
& c_{10} & {[0.0,1.0]} \\
& c_{11} & {[0.0,1.0]} \\
& c_{12} & {[0.0,1.0]} \\
& c_{13} & {[0.0,1.0]} \\
& c_{14} & {[0.0,1.0]} \\
& c_{15} & {[0.0,1.0]} \\
& c_{16} & {[0.0,1.0]} \\
& c_{17} & {[0.0,1.0]} \\
c_{18} & {[0.0,1.0]} \\
c_{19} & {[0.0,1.0]} \\
c_{20} & {[0.0,1.0]} \\
c_{21} & {[0.0,1.0]}
\end{array}\right] .
$$

Get the section filed $R_{p}$ according to the value region of the evaluating element in the Tables 1 and 3.

Step 3. Obtain the relation function values $K\left(x_{i j}\right)$ of the evaluation indices by formula (6) in Table 3.

Step 4. Obtain the weight values of the evaluation indices by formula (8) in Table 3.

Step 5. Obtain the synthetic relating degree and the result of evaluation.

Calculated by (5) and (6), the synthetic relating degree of the urban road traffic safety in three cities is shown in Table 4

The evaluation results in Table 4 show that the road traffic safety of the third city, Changzhou city, belongs to Level 2, and that city, however, is basically sustainable developing. Urban road traffic safety of the second city, Hefei city, belongs to Level 4, meaning that the sustainable development is close to the extremity. It is important for the second city, Changzhou city, to find new methods and advanced management technologies to develop urban traffic system and hence increase sustainable development capacity. In the same manner, 
the first city, Zhenjiang city, belongs to Level 3. Therefore it is concluded that road traffic safety is basically rational and basically sustainable developing in one city. However, we should raise sustainable development level for road traffic safety in one city, accelerate intelligence and information for urban traffic system, and promote coordinated development in entire city in future plan.

\section{Conclusions}

In this paper the urban road traffic safety evaluation problems have been studied. An objective-evaluating model has been developed to determine the grade of the safety of the city, as well as a practical approach to rank the safety of the city. Based on the concept of matter element, this paper offers a new method to solve the traffic safety problems through interval numbers. Emphasis was placed on the construction of a criterion function by which traffic safety achieved a hierarchical system of objectives to be evaluated. The matter element matrix solves the uncertainty and incompatibility of the evaluated factors used to assess urban road traffic safety. The application results showed that the new model is more reasonable and persuasive.

Conclusions and future work are summarized as well. The proposed method provides a relatively objective basis for the short-term and long-term planning of the protection of urban resources and creates a foundation for the traffic management and urban traffic sustainable development. Moreover, it provides a scientific basis for the policy decision of urban traffic construction and a train of new thought for evaluated method in this field. It is necessary for urban road to adopt advanced technologies to increase the safety.

\section{Conflict of Interests}

The authors declare that there is no conflict of interests regarding the publication of this paper.

\section{Acknowledgments}

The authors are very grateful to the anonymous referees for their insightful and constructive comments and suggestions that have led to an improved version of this paper. The work was supported by National nature science funding of China (Project no. 51178157).

\section{References}

[1] W. Wang, W. Zhang, H. Guo, H. Bubb, and K. Ikeuchi, "A safetybased approaching behavioural model with various driving characteristics," Transportation Research C, vol. 19, no. 6, pp. 1202-1214, 2011.

[2] J. Šaparauskas and Z. Turskis, "Evaluation of construction sustainability by multiple criteria methods," Technological and Economic Development of Economy, vol. 12, no. 4, pp. 321-326, 2006.
[3] O. Taubman, B. Ari, and E. Shay, "The association between risky driver and pedestrian behaviors: the case of Ultra-Orthodox Jewish road users," Transportation Research F, vol. 15, no. 2, pp. 188-195, 2012.

[4] E. K. Zavadskas, "Multi-attribute assessment of road design solutions by using the COPRAS method," The Baltic Journal of Road and Bridge Engineering, vol. 2, no. 4, pp. 195-203, 2007.

[5] A. Ceder, Public Transit Planning and Operation-Theory, Modelling and Practice, chapter 10, Elsevier, Oxford, UK, 2007.

[6] I. A. Kaysi and A. S. Abbany, "Modeling aggressive driver behavior at unsignalized intersections," Accident Analysis and Prevention, vol. 39, no. 4, pp. 671-678, 2007.

[7] O. Lingaitiene, "A mathematical model of selecting transport facilities for multimodal freight transportation," Transport, vol. 23 , no. 1, pp. 10-15, 2008.

[8] H. W. Warner, T. Özkan, T. Lajunen, and G. Tzamalouka, "Cross-cultural comparison of drivers' tendency to commit different aberrant driving behaviours," Transportation Research $F$, vol. 14, no. 5, pp. 390-399, 2011.

[9] I. Jaržemskiene, "The evolution of intermodal transport research and its development issues," Transport, vol. 22, no. 4, pp. 296-306, 2007.

[10] W. K. M. Brauers, E. K. Zavadskas, F. Peldschus, and Z. Turskis, "Multi-objective decision-making for road design," Transport, vol. 23, no. 3, pp. 183-193, 2008.

[11] Q. Hu and H. Lu, "Urban ecological traffic evaluation based on matter element analysis," in Proceedings of the International Conference on Intelligent Computing and Integrated Systems (ICISS '10), pp. 573-576, Guilin, China, October 2010.

[12] L. I. Yanguo, "Study on tourist carrying capacity based on matter element analaysis," Ecological Econimy, vol. 1, no. 2, pp. 23-27, 2005.

[13] S.-T. Wang and M.-H. Li, "Analysis of cost models and quantification models of customisation degree after VMI strategy implemented in different industries," International Journal of Manufacturing Technology and Management, vol. 26, no. 1-4, pp. 161-175, 2012.

[14] Q. Hu and W. Deng, "Comprehensive evaluation for the traffic safety of the expressway based on quantitative analysis," in Proceedings of the 1st International Conference on Transportation Engineering (ICTE '07), pp. 680-685, July 2007.

[15] J. L. Zhang and X. Y. Wang, "Extraction and dynamic deduction method of vehicle driving tendency feature under time variable free flow condition," Journal of Beijing Institute of Technology, vol. 20, no. 1, pp. 127-133, 2011.

[16] Y. J. Yang, Y. P. Wang, and X. M. Zhao, "Research on staff scheduling of urban passenger taxi dispatching center based on genetic algorithm," Journal of Highway and Transportation Research and Development, vol. 27, no. 7, pp. 142-146, 2010.

[17] Y. Q. Feng and C. Q. Fang, "Cluster analysis of drivers characteristics evaluation," Communications Science and Technology Heilongjiang, no. 11, pp. 161-163, 2007.

[18] S. Vukmirovic, A. Erdeljan, I. Lendak, D. Capko, and N. Nedic, "Optimization of workflow scheduling in utility management system with hierarchical neural network," International Journal of Computational Intelligence Systems, vol. 4, no. 4, pp. 672-679, 2011. 
[19] Y. Y. Zhang, X. Y. Wang, and J. L. Zhang, "Verification method of vehicle driving tendency recognition model under free flow," Journal of Computer Application, vol. 32, no. 2, pp. 578-580, 2012.

[20] H. M. Niu, "Determination of the skip-stop scheduling for a congested transit line by bilevel genetic algorithm," International Journal of Computational Intelligence Systems, vol. 4, no. 6, pp. 1158-1167, 2011. 

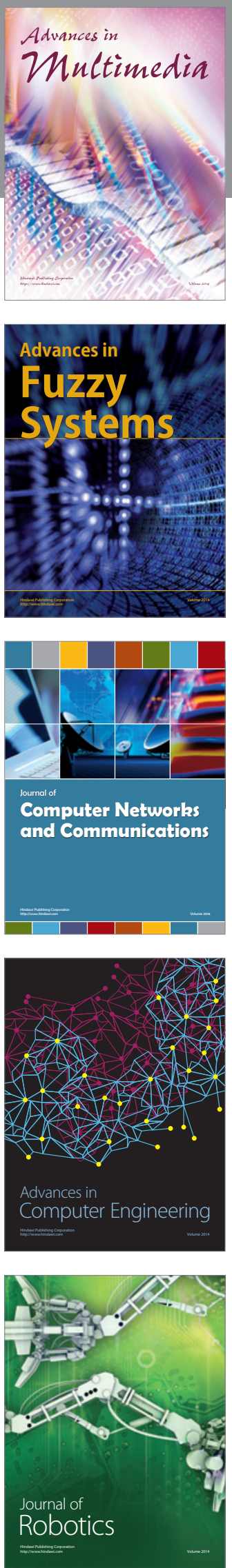

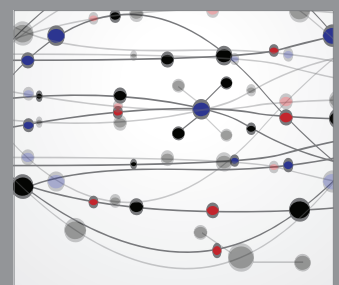

The Scientific World Journal
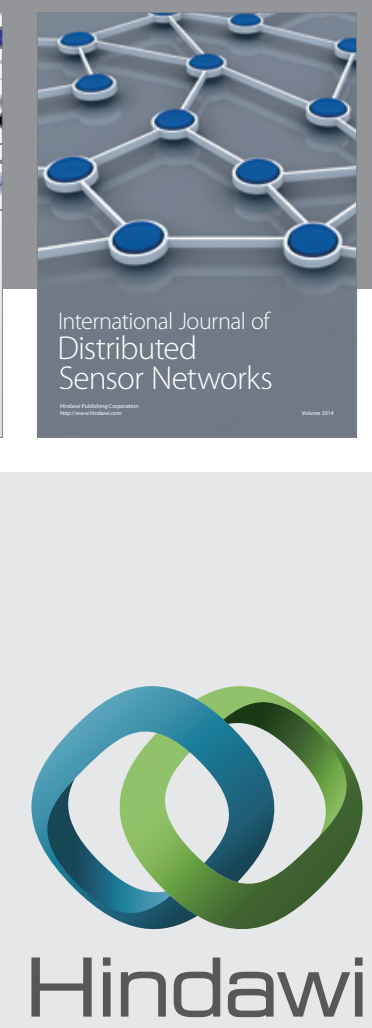

Submit your manuscripts at

http://www.hindawi.com
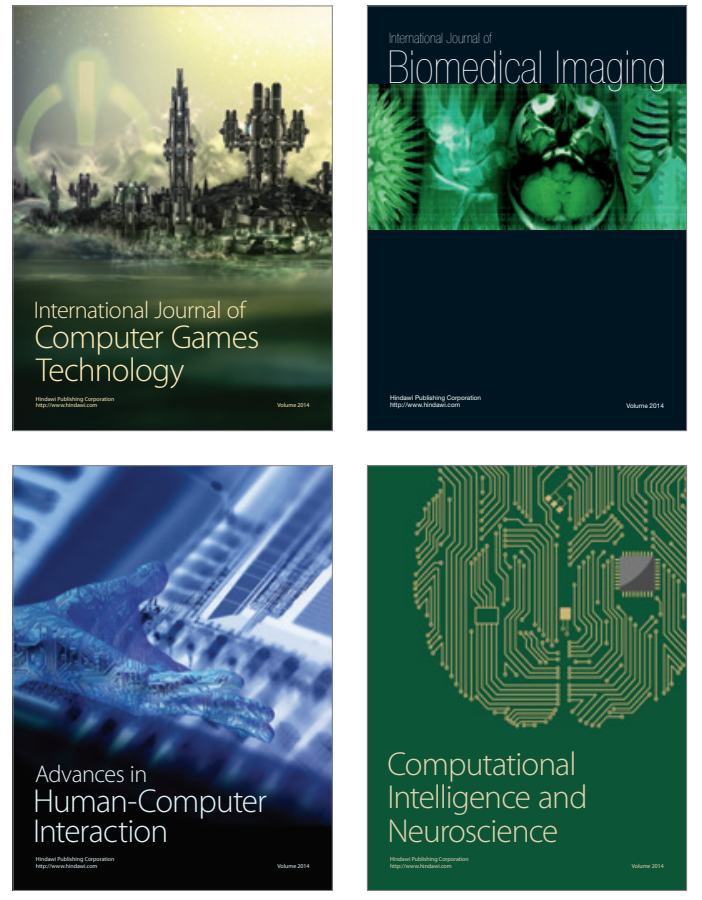
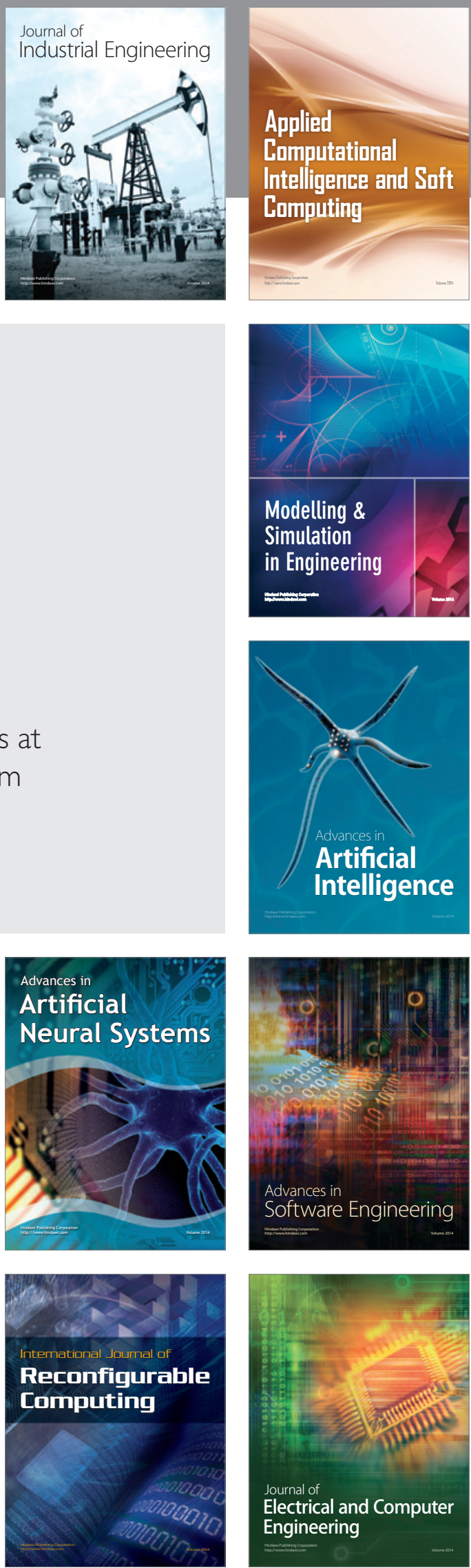